\title{
El soporte nutricional en la situación de enfermedad terminal. Dilemas éticos
}

\author{
A. AZULAY TAPIERO, E. HORTELANO MARTÍNEZ
}

Servicio de Medicina Interna. Hospital Dr. Moliner. Serra, Valencia

\section{RESUMEN}

La malnutrición es un problema frecuente en la situación de enfermedad terminal. La indicación de un soporte nutricional artificial en estos pacientes crea conflictos éticos de difícil solución.

En este trabajo se sugieren líneas de actuación sencillas que puedan servir de orientación clínica, si bien la toma de decisiones debe ser siempre individualizada, teniendo en cuenta el balance entre riesgos y beneficios de esta modalidad de tratamiento.

Se debe dar una máxima prioridad a los deseos del paciente

PALABRAS CLAVE: Soporte nutricional. Enfermedad terminal. Ética.
NUTRITIONAL SUPPORT IN TERMINALLY ILL SITUATION. ETHICAL DILEMMAS

\begin{abstract}
Malnutrition is a common complication in terminally ill situation. Artificial nutritional support in these patients create a host of ethical conflicts with difficult solutions.

In this paper we give simple suggestions that might the help care provider in making a viable clinical decision. However, each case should be treated in an individualized manner taking into consideration the ratio between risks and benefits of this treatment.

The patient's wishes should have the highest priority.
\end{abstract}

KEY WORDS: Nutritional support. Terminally Ill. Ethics.

Azulay Tapiero A, Hortelano Martínez E. El soporte nutricional en la situación de enfermedad terminal. Dilemas éticos. An Med Interna (Madrid) 2003; 20: 434-437

\section{INTRODUCCIÓN}

El soporte nutricional constituye una modalidad de tratamiento aceptada desde el punto de vista médico, moral y legal en la totalidad de pacientes con alguna esperanza, aunque sea mínima, de supervivencia.

La imposibilidad para conseguir un aporte nutritivo aceptable constituye un factor de mal pronóstico en la situación de enfermedad terminal y produce un gran impacto emocional en el enfermo y en su familia. Después de la disnea, la falta de ingesta es el problema que más distrés físico y emocional produce en los pacientes diagnosticados de cáncer; el dolor ocuparía el tercer lugar (1).

La anorexia y la pérdida de peso que aparecen en la situación de enfermedad terminal suelen tener un origen multifactorial, en donde están implicados mediadores químicos (citoquinas), la presencia de otros síntomas, efectos colaterales de los tratamientos administrados, infecciones, factores mecánicos, metabólicos, hormonales, psicológicos, etc.
En la gran mayoría de los casos no es posible un tratamiento causal de este problema, por lo que la terapéutica debe basarse fundamentalmente en el consejo dietético y en el uso de unos agentes farmacológicos no siempre efectivos (acetato de megestrol y corticoides fundamentalmente) en algunos casos de anorexia, en la utilización de sistemas artificiales de nutrición enteral o colocación de prótesis autoexpandibles ("stents") en casos de disfagia o síndromes de oclusión intestinal, o bien hacer uso de la nutrición parenteral cuando hay inutilidad del tubo digestivo. Un arma terapéutica fundamental y a aplicar en todos los casos será hacer que el enfermo y su familia se adapten al problema, haciendo uso de una comunicación adecuada.

\section{DISCUSIÓN}

El soporte nutricional artificial debe formar parte de los cuidados paliativos integrales siempre que su objetivo sea

Trabajo aceptado: 5 de febrero de 2003 
conseguir o mantener el estado de bienestar del paciente; en otros supuestos, su aplicación podrá ser más o menos discutible, dependiendo del estado del enfermo y de sus preferencias, de tal modo que el soporte nutricional podría considerarse una modalidad de tratamiento agresiva o desproporcionada en determinados casos, constituyendo incluso una forma de obstinación o ensañamiento terapéutico, totalmente inaceptable desde el punto de vista ético.

En la situación de enfermedad terminal, mantener la nutrición y la hidratación, siempre que la vía oral sea útil, que no suponga un gran esfuerzo para el enfermo y que no se requiera el uso de métodos artificiales más o menos agresivos para su aplicación, deberá considerarse siempre una actitud correcta y una obligación ética, por más deteriorado que esté el paciente y por más próxima que se prevea la muerte. La nutrición y la hidratación, en estos supuestos, forman parte de los cuidados humanitarios básicos o mínimos, nunca desproporcionados y, como tales, deben ser aplicados.

El soporte nutricional enteral mediante métodos artificiales, nos referimos fundamentalmente al sondaje nasogástrico o a la gastrostomía, en el contexto en el que nos movemos, en ningún caso va a curar la enfermedad ni va a evitar la progresión hacia la muerte, pero podría mejorar la calidad de vida, parámetro que debe ser siempre subjetivo, podría evitar complicaciones y hacer que el deterioro sea menos rápido; en estos casos, que se dan con más frecuencia en tumores de cabeza y cuello (2), siempre que se cuente con el consentimiento del enfermo, lo éticamente correcto sería aplicarlo.

La nutrición parenteral, en la situación de enfermedad terminal, cuando se ha descartado cualquier tratamiento con finalidad curativa, debe usarse de manera muy excepcional y siempre con el consentimiento del enfermo bien informado, ya que constituye una técnica agresiva, que limita mucho la autonomía del enfermo y que está sujeta a multitud de complicaciones, fundamentalmente de tipo infeccioso, metabólico y las derivadas de la colocación de un catéter por vía central. En algunos casos de obstrucción intestinal, cuando se estima una expectativa de vida relativamente prolongada, la nutrición parenteral podría mejorar el estado del enfermo y por tanto estar excepcionalmente indicada (2).

Cuando no se dan estos requisitos, cuando el paciente es incapaz de alimentarse por si mismo, cuando la anorexia es intensa y no responde a ningún tratamiento y cuando cualquier sistema de nutrición artificial no mejora su calidad de vida sino que la empeora, aparece el dilema y la controversia, sobre todo cuando se hace necesario la utilización de medidas artificiales como el sondaje nasogástrico o la gastrostomía endoscópica percutánea como posible solución al problema.

Si finalmente se ha decidido instaurar un sistema de nutrición artificial, pueden aparecer dos nuevas causas de conflicto ético:

- Al considerar la posibilidad de retirarlo cuando se ha mostrado ineficaz, cuando produce más perjuicios que beneficios y cuando la enfermedad progresa de forma inevitable hacia la muerte a pesar de su aplicación.

- Cuando se hace necesario utilizar dispositivos de restricción física o sedación farmacológica para evitar la autodesintubación, situación que se da con relativa frecuencia en caso de deterioro cognitivo importante (demencia o delirio), en donde, obviamente, no puede aplicarse el principio ético de autonomía.

Con respecto al primer supuesto, conviene aclarar que, desde el punto de vista ético, tiene el mismo valor no iniciar un tratamiento que interrumpirlo cuando ya no se considera necesario o útil; incluso se podría considerar que la negativa a iniciar un tratamiento puede ser moralmente menos aceptable, ya que, en ese momento, se suele disponer de menos información del proceso patológico que cuando, tras observar la evolución de la enfermedad, se decide retirarlo (3); pero, aunque moralmente pueda ser lo mismo no iniciar que retirar una técnica de soporte vital, en la práctica diaria es mucho más difícil optar por la segunda opción, y más si la muerte sigue a la interrupción del tratamiento, lo que podría establecer una relación causa-efecto con todas las implicaciones éticas, religiosas e incluso legales que ello podría acarrear, por lo que requerirá, la mayoría de las veces, de una reflexión prudente por parte del equipo multidisciplinar, valorando muy bien la relación entre perjuicios y beneficios que supone el seguir aplicándola y llegando a un consenso con el enfermo y sus familiares que deben estar en todo momento correctamente informados de la situación para que puedan participar en la toma de decisiones.

Cuando ocurre el segundo supuesto, en la situación de enfermedad terminal, debe quedar claro que las desventajas del soporte nutricional artificial superan a los beneficios y más si tenemos en cuenta que la restricción física, o más claramente "atar al enfermo", viola los fundamentos básicos de los cuidados humanitarios y la dignidad de la persona. Estas medidas sólo deberían utilizarse cuando la alimentación por sonda nasogástrica sea imprescindible para el sostén de la vida durante una enfermedad aguda y reversible, no para dicho sostén en casos de padecimientos irreversibles y con nulas posibilidades de recuperación de la enfermedad o la consecución de la independencia alimentaria (4).

En resumen, los dilemas bioéticos que surgen cuando se plantea la necesidad de utilizar un sistema artificial de soporte nutricional, aparte de plantear conflictos entre los principios de beneficencia, no-maleficencia y autonomía, se basan fundamentalmente en la calificación moral que se da a la nutrición en estas circunstancias patológicas, planteándose la cuestión de si debe considerarse a la nutrición artificial como un acto médico, y como tal debe tener sus indicaciones y sus contraindicaciones, o en cambio es un acto humanitario básico y elemental que se tiene que aplicar en cualquier circunstancia. Si se considera a la nutrición artificial como un acto humanitario básico a aplicar en cualquier circunstancia, en principio, no surgirá ningún conflicto ético, se instaura siempre que exista incapacidad para la nutrición por vía oral y asunto resuelto; sin embargo, si se considera un acto médico, estará sujeto a múltiples conflictos, a veces de difícil solución.

La tendencia a considerar a la nutrición y a la hidratación como terapias que proporcionan bienestar y eliminan el sufrimiento en todos los casos, por tanto siempre moralmente justificadas, se basa en nuestras propias experiencias cuando tenemos la sensación de sed o hambre, asociamos la falta de ingesta con estas dos sensaciones; pero si consideramos la evidencia, se debe reevaluar esta justificación, ya que el bienestar y la eliminación del sufrimiento podrían quedar en entredicho (5). Hay que tener en cuenta que el beneficio del soporte nutricional, al menos en pacientes oncológicos en situación terminal, es muy dudoso (6) y difícilmente revierte la desnutrición ya existente, no mejora la supervivencia y no evita las complicaciones (7-9); conclusiones similares se han visto en pacientes con demencia avanzada, en donde la alimentación con sonda nasogástrica no se ha mostrado eficaz en 
la prevención de neumonía por aspiración, no ha conseguido prolongar la supervivencia, ni ha reducido el riesgo de desarrollar úlceras por presión (10).

En el mejor de los casos, en la demencia muy evolucionada, lo único que podría conseguir la aplicación de un soporte nutricional artificial sería aumentar la cantidad de vida, pero a costa de un empeoramiento de su calidad y un aumento del número e intensidad de las complicaciones; el paciente podrá vivir más tiempo, pero en unas condiciones de gran sufrimiento por la presencia de complicaciones que, dejando que la enfermedad siga su curso, no tendrían que aparecer $(9,11)$.

Los dilemas bioéticos que pueden surgir en torno a la nutrición artificial, en el enfermo en situación terminal, no deben centrarse en la calidad o en la cantidad de los nutrientes, sino en su indicación y en su eficacia (12).

Es frecuente que la familia crea que el deterioro del enfermo es debido a la mala nutrición, por lo que es importante tener bien claro desde el principio, y así lo debemos dar a entender al enfermo y a su familia, que la falta de ingesta que se produce en esta situación es una consecuencia de la enfermedad, que es "normal" su presencia en los estados terminales avanzados y que no es la causa de este deterioro rápido y progresivo que presenta el enfermo.

Las decisiones apropiadas sobre la utilización de cualquier forma de soporte vital, incluidas la nutrición y la hidratación, requieren una prudente reflexión por parte del equipo multidisciplinar y del comité de bioética (13), si es que existe. También sería conveniente la presencia del dietista en dicho equipo multidisciplinar, que realizaría una labor importante en la planificación del soporte nutricional para el paciente capaz de decidir y en la ayuda que debe prestar a la familia para que se adapte lo mejor posible a la situación y pueda tomar la mejor decisión cuando el paciente no puede ejercer su autonomía y la muerte está próxima $(13,14)$.

No aplicar un soporte nutricional no es una opción que por si sola sea suficiente para conseguir una buena calidad de muerte; en muchas ocasiones será necesario utilizar otras medidas paliativas, algunas de ellas con la intención de producir una disminución profunda e irreversible del nivel de conciencia, lo que planteará un dilema ético importante al ser, en estos casos, imprecisos los límites que separan una actitud "pasiva" para conseguir una muerte digna y una forma de eutanasia activa (15).

Es indispensable, para actuar correctamente, conocer cuáles son los valores del enfermo y su opinión acerca de los cuidados de su salud, su nutrición y otras necesidades sanitarias, para que, dentro de lo posible y conforme a la legislación vigente, la atención que reciba sea planificada y llevada a cabo de acuerdo con sus deseos. Los pacientes tienen derecho a rechazar cualquier tratamiento, incluidos los de soporte vital, y la violación de este derecho puede ser sancionable desde el punto de vista legal. Si el paciente es incapaz de tomar decisiones en el momento determinado, el "testamento vital" o las "directrices anticipadas" podrán ser los únicos indicadores de estos valores. En el caso de que el paciente sea incompetente y no se disponga de instrucciones anticipadas, la familia deberá expresar lo que sabe acerca de los deseos y valores del enfermo y se deberá actuar en consonancia con ellos. En ausencia de familiares, amigos o tutores, el equipo multidisciplinar deberá tomar la decisión que considere más adecuada sobre la base de los intereses del paciente.

A pesar de que el principio de autonomía debe ser fundamental a la hora de aplicar o no una técnica de soporte vital, existen evidencias que demuestran que la elección completamente libre es un ideal que se da muy pocas veces y que, por parte del personal sanitario, es muy difícil librarse de ejercer una cierta coacción, siempre con buena intención. El paciente y/o sus familiares suelen aceptar lo que el personal sanitario, fundamentalmente el médico, sugiere (16), por lo que los valores y las creencias de los miembros del equipo asistencial también tienen mucho que ver con la decisión final que se toma, sobre todo en temas tan conflictivos como la nutrición y la hidratación en los que aún no hay un consenso sobre si constituyen una modalidad de tratamiento, con sus indicaciones y contraindicaciones, o una necesidad básica que debe suministrarse en cualquier circunstancia.

\section{CONCLUSIONES}

El soporte nutricional, en la situación de enfermedad terminal, cuando la muerte es una certeza absoluta sea cual sea nuestra actuación, debe aplicarse con el objetivo de mejorar la calidad de vida.

Es fundamental contar con la opinión del paciente bien informado, o de su representante en caso de incapacidad, para encontrar la opción más adecuada. Debe evitarse la coacción por parte del personal sanitario.

Quizás sea en el marco de unos Cuidados Paliativos de máxima calidad el lugar más adecuado para tratar a este tipo de pacientes, ya que, además de brindar una atención integral al enfermo y a su familia para que sean cubiertas todas sus necesidades, su filosofía se basa en la aplicación sistemática de los principios bioéticos fundamentales: beneficencia, no maleficencia, autonomía y justicia (17).

\section{Bibliografía}

1. Portenoy RK, Thaler HT, Kornblith AB, Lepore JM, Friedlander-Klar $\mathrm{H}$, Coyle N, et al. Symptom prevalence, characteristics and distress in a cancer population. Qual Life Res 1994; 9: 183-9.

2. Bachmann P, Marti-Massoud C, Blanc-Vincent MP, et als. Standards, options and recommendations: nutritional support in palliative or terminal care of adult patients with progressive cancer. Bull Cancer 2001; 88 (10): 985-1006.

3. Azulay A. Dilemas bioéticos en la situación de enfermedad terminal y en el proceso de la muerte. Med Pal 2000; 7: 145-56.
4. Quill TE: Utilization of nasogastric feeding tubes in a group of chronically ill elderly patients in a comunity hospital. Arch Intern Med 1989; 149: 1937.

5. Winter SM. Terminal nutrition: framing the debate for the withdrawal of nutritional support in terminally ill patients. Am J Med 2000; 109: 740-1.

6. Dresler CM, Jeevanandam M, Brennan MF. Metabolic efficacy of enteral feeding in malnoutrished cancer and non cancer patients. Metabolism 1987; 36: 82-8. 
7. McCann RM, Hall WJ, Groth-Juncker A. Comfort care of terminally ill patients. The appropiate use of nutrition and hydration. JAMA 1994; 272: 1263-6.

8. Torelli GF, Campos AC, Meguid MM. Use of TPN in terminally ill cancer patients. Nutrition 1999; 15: 665-7.

9. Finucane TE, Christmas C, Travis K. Tube feeding in patients with advenced dementia: a review of the evidence. JAMA 1999; 282: 136570.

10. Douglas DR, Carla SA. Management of common symptoms in terminally ill patients: Part I. Fatigue, anorexia, cachexia, nausea and vomiting. Am Fam Physician 2001; 64: 807-14.

11. Gillick MR. Rethinking the role of tube feeding in patients with advanced dementia. N Engl J Med 2000; 342: 206-10.

12. Klein S, Kinney J, Jeejeebhoy K, Alpers D, Hellerstein M, Murray M.
Nutrition support in clinical practice: review of published data and recommendations for future research directions. J PEN 1997; 21: 13356.

13. Brody H, Noel MB. Dietitians' role in decisions to withold nutrition and hydration. J Am Diet Assoc 1991; 91: 580-5.

14. Boisaubin EV. Ethical issues in the nutritional support of the terminal patient. J Am Diet Assoc 1984; 84: 529-31.

15. Englert M, Ronson A, Lossignol D, Body JJ. Terminal medical interventions: psychosocial, medical, ethical and legal aspects. Rev Med Brux 2001; 22 (2): 93-9.

16. Bruera E, MacDonald N. To hydrate or not hydrate: how should it be? Journal of Clinical Oncology 2000; 18: 1156-8.

17. Azulay A. Los principios bioéticos: ¿se aplican en la situación de enfermedad terminal? An Med Interna (Madrid) 2001; 18: 650-4. 